\title{
Assessment of cardiac autonomic parameters in patients with uncomplicated familial Mediterranean fever
}

\author{
(1) Mustafa Candemir ${ }^{1}$, (1) Burak Sezenöz $z^{1}$, (1) Burcu Candemir ${ }^{2}$, (1) Serdar Gökhan Nurkoç³ \\ 1 Gazi University Faculty of Medicine, Department of Cardiology, Ankara, Turkey \\ 2 Yozgat City Hospital, Clinic of Internal Medicine, Yozgat, Turkey \\ 3Yozgat City Hospital, Clinic of Cardiology, Yozgat, Turkey
}

\section{Date submitted:}

22.07.2021

Date accepted:

25.10.2021

Online publication date:

15.03.2022

\section{Corresponding Author:}

Mustafa Candemir, M.D., Gazi

University Faculty of Medicine,

Department of Cardiology, Ankara,

Turkey

mstfcndmr@hotmail.com

ORCID:

orcid.org/0000-0002-3645-3912

Keywords: Cardiac autonomic dysfunction, familial Mediterranean fever, heart rate turbulence, heart rate variability, Tp-e, Tp-e/QTc

\begin{abstract}
Aims: Cardiac dysautonomia and arrhythmias can occur in chronic inflammatory conditions such as familial Mediterranean fever (FMF). The aim of this study was to evaluate the cardiac autonomic functions in patients with FMF.

Methods: This was a retrospective case-control study. Electrocardiography (ECG) and 24-hour Holter ECG recordings were obtained from the medical records. T-peak to T-end intervals (Tp-e) and Tp-e/corrected QT (QTc) ratio were calculated on ECG. Heart rate variability (HRV) parameters and heart rate turbulence (HRT) parameters including turbulence onset (TO) and turbulence slope (TS) were evaluated using the 24-hour Holter ECG. All subjects were divided into three groups according to HRT parameters (HRT-0: normal TO and TS; HRT-1: abnormal TS or TO; and HRT-2: abnormal TO and TS).
\end{abstract}

Results: The study included 115 individuals (age, years, mean \pm standard deviation: $34.5 \pm 9.6$ years, $57 \%$ female, $61 \mathrm{FMF}$ patients and 54 controls). Patients with FMF exhibited an increased ratio of Tp-e, Tp-e/QTc ratio ( $p<0.001$ for both), and impairment in all HRV parameters $(\mathrm{p}<0.05$ for all). Disease duration was correlated with Tp-e $(r=0.402, p=0.001), T p-e / Q T c$ ratio $(r=0.382$, $p=0.003$ ) and HRV parameters ( $r>0.264, p<0.05$ for all). Of patients with FMF, 32 (52.5\%) were in the HRT-1 group, and 9 (14.8\%) were in the HRT-2 group. Patients using anakinra showed more impairment in the HRV parameters ( $p<0.05$ for all) and Tp-e/QTc ratio $(p=0.041)$ compared to patients who were not on anakinra.

Conclusions: This study showed cardiac autonomic system impairments in patients with FMF without complications, which correlated with the disease duration.

\section{Introduction}

Familial Mediterranean fever (FMF) is a chronic inflammatory disease that is usually inherited as an autosomal recessive disorder $(1,2)$. Although it is a widespread disease, some ethnic groups, such as Turks, Arabs, and Hebrews, have a higher prevalence $(1,3)$. FMF is characterized by recurrent attacks of fever, accompanied by abdominal and joint pain (2). Besides these clinical features, chronic inflammation and amyloid deposits have been linked to significant cardiovascular complications such as pericarditis, atherosclerosis, cardiomyopathy, and pulmonary hypertension (4). Colchicine is recommended to reduce attacks and prevent complications (5). In recent studies, anakinra, an interleukin-1 (IL-1) receptor antagonist, is effective and safe in colchicine-resistant patients (6).

The cardiac autonomic nervous system (ANS) is crucial in the regulation of the cardiovascular system. Regardless of the underlying disease, cardiac autonomic dysfunction can cause ventricular arrhythmias, which can lead to sudden death (7). Cardiac arrhythmias, which are associated with poor prognosis, can occur in chronic inflammatory diseases such as FMF due to cardiac dysautonomia (8). Chronic inflammation can cause 
adverse cardiovascular outcomes such as atrial fibrillation and ventricular arrhythmias (9). Cardiometabolic events are also one of the leading causes of death in chronic inflammatory diseases (10).

Cardiac ANS functions can be evaluated using several non-invasive methods. One of these is the 24-hour Holter electrocardiography (ECG), which is a simple and practical method. Heart rate variability (HRV) and heart rate turbulence (HRT) are parameters in 24-hour Holter ECG that provide information about the cardiac ANS (11). ECG examination is another simple method to evaluate cardiac ANS. The measurement of the time course for the repolarization of myocardial Moe cells, which is believed to be related to cardiac arrhythmia, has recently been reported to be a valuable tool for evaluating cardiac ANS (12). This measurement is recorded at resting ECG and is defined as the peak-to-end interval of the T wave (Tp-e). Prolonged Tp-e and Tp-e/corrected QT interval (QTc) ratios, as well as aberrant HRV and HRT parameters, were associated with malignant ventricular arrhythmias and cardiac death (13).

Some studies found that cardiac autonomic functions were impaired in FMF patients without amyloidosis, while others found the opposite $(14,15)$. This study investigated whether the cardiac ANS is impaired in FMF patients by evaluating the 24hour Holter parameters as well as the Tp-e and Tp-e/QTc ratio on the ECG.

\section{Methods}

\section{Study population}

This retrospective case-control study reviewed the records of 61 patients with FMF and 54 control subjects who presented to our cardiology outpatient clinic for any reason between April 2019 and January 2020 and underwent a 24-hour Holter record. In the FMF group's hospital records, there was no history of FMF attacks in the last 3 months preceding the 24-hour Holter ECG. We excluded control patients who had some form of cardiac disease or comorbidity. Those without a suitable 24hour Holter ECG recording (with artifacts and $<5$ premature ventricular complexes) and those without an appropriate ECG recording (with $\mathrm{T}$ negativity, biphasic $\mathrm{T}$ waves, and $\mathrm{U}$ waves) were excluded from the study. Additionally, patients with a history of documented diabetes mellitus, hypertension, coronary artery disease, atrial fibrillation or rhythm abnormalities, additional chronic inflammatory diseases, active infections, malignancies, or diagnosed with amyloidosis, structural heart disease, proteinuria in 24-hour urine test (to exclude amyloidosis), as well as those using medications known to affect cardiac autonomic function (antidepressants, b-blockers, calcium channel blockers, antiarrhythmics), were excluded. In our hospital, where the study was conducted, the number of patients using biological agents other than anakinra was very low. Therefore, only the patients who used anakinra were included in the study, and the patients who used other biological agents were excluded.

The Local Ethics Committee approved the study (Yozgat BozokUniversity, approval no:2017-KAEK-189_2021.04.28_10), and its protocol conformed to the principles of the Declaration of Helsinki.

\section{ECG examination}

Medical records were used to produce resting ECG recordings with an amplitude of $10 \mathrm{mV}$ at a rate of $25 \mathrm{~mm} / \mathrm{sec}$. All of the ECG recordings were transferred to a computer and measurements of the peak-to-end interval of the $\mathrm{T}$ wave (Tpe) were performed from the precordial leads. An average value of two readings was calculated for each lead. The following ventricular repolarization parameters were calculated: QT interval, QT interval corrected for heart rate, and Tp-e (Figure 1). The results of standard transthoracic echocardiography and 24-hour Holter monitoring (GE medical systems information technologies, Inc., software version 8.0.3, Milwaukee, USA) with 3-lead analog devices were evaluated independently by two different cardiologists.

\section{Heart rate variability and heart rate turbulence}

Holter monitoring recordings were exported to the computer and manually scanned to remove artifacts and analyzed with the Holter software. HRV and HRT parameters were automatically documented by the Holter software as numerical data.

In HRV analysis were obtained time-domain parameters, including the standard deviation (SD) of all NN intervals for a given period (SDNN), SD of the 5 min mean R-R intervals tabulated over an entire day (SDANN), square root of the mean of the sum of the squares of differences between adjacent $\mathrm{R}-\mathrm{R}$ intervals ( $\mathrm{MSSD}$ ), and the proportion of differences in successive NN intervals greater than $50 \mathrm{~ms}$ (pNN50) as well as frequency domain parameters, including high frequency (HF) component (0.15-0.40 Hz), low frequency (LF) component

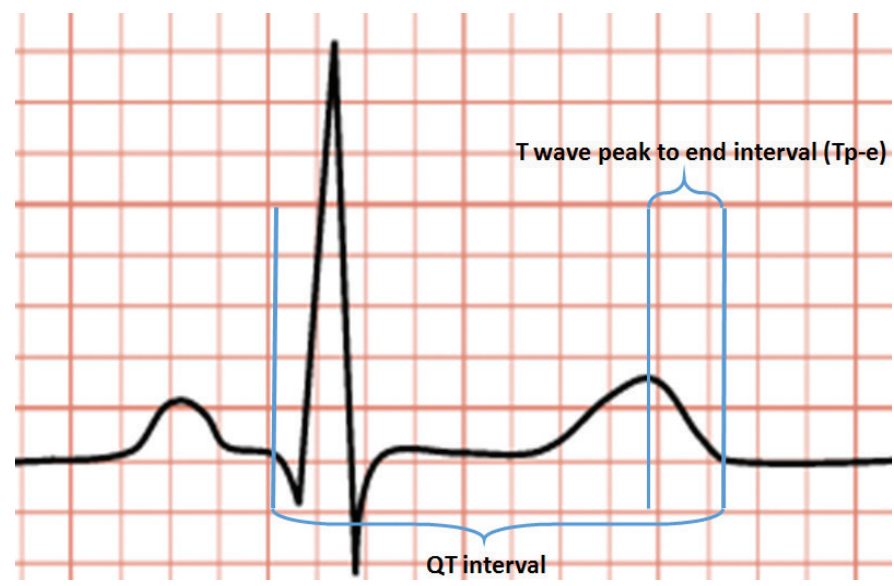

Figure 1. Demonstration of the T wave peak to end and QT intervals 
(0.04-0.15 Hz) and very LF (VLF) component $(0-0.04 \mathrm{~Hz})$ and LF/HF ratio (16).

HRT analysis was carried out by an automatic computer program. To minimize false evaluations, patients having at least five ventricular extrasystoles were included in the assessment. The two phases of HRT were numerically quantified as turbulence onset (TO) and turbulence slope (TS). TO was defined as the percentage change in the RR intervals following premature ventricular beats (PVBs) versus (vs.) the pre-PVB period. TO represents the initial acceleration of heart rate following PVB. TO $\leq 0 \%$ (negative TO) whereas TS $>2.5 \mathrm{~ms} / \mathrm{RR}$ (positive TS) were accepted as normal values. The study population was separated into three groups based on their HRT data. HRT-0 means that both values are within normal limits, HRT-1 implies that one of the two parameters is abnormal and HRT-2 means both parameters are abnormal (17).

\section{Statistical Analysis}

The IBM Statistical Package for the Social Sciences for Windows, version 21.0, IBM.Corp., Armonk, NY, 2012 was used for the statistical analysis. The Kolmogorov-Smirnov test was used to test the normality of distribution. Continuous variables were presented as mean $\pm S D$, and categorical variables were expressed as a number and a percentage (\%). The Student's t-test or the Mann-Whitney $U$ test was used to compare continuous variables. The chi-square test or Fisher's exact test was used to compare categorical variables. Pearson's correlation test was used for the normally distributed data, and Spearman's correlation test was used for non-normally distributed data. A two-sided $p<0.05$ was considered significant.

\section{Results}

A total of 115 individuals were included in the study. The demographic characteristics of the patients ( $n=61,43$ females; mean $\pm S D, 34.3 \pm 10.0$ years) and the control group $(n=54,33$ females; mean $\pm S D, 34.7 \pm 9.3$ years) are presented in Table 1 . There were no significant differences between the two groups in terms of age, gender, smoking, or body mass index. The median disease duration was 7.0 (3.0-10.5) years. All patients were on colchicine (1-1.5 mg/day); however, eight patients were receiving anakinra+colchicine $(1.5-2 \mathrm{mg} /$ day $)$ due to colchicine resistance. The median duration of anakinra treatment was 45 (19.5-69) months. The most common mutations seen in patients were M694V ( $n=35 ; 57.3 \%), \operatorname{M680I}(n=8 ; 13.1 \%), \operatorname{V726A}(n=7$; $11.4 \%)$, and $\mathrm{E} 148 \mathrm{Q}(\mathrm{n}=6 ; 9.8 \%)$. There was no mutations in six patients $(9.8 \%)$. Of the 55 patients with mutations, $18(29.5 \%)$ were heterozygous, $22(37.7 \%)$ were compound heterozygous, and $15(22.9 \%)$ were homozygous. ECG and 24-hour Holter ECG parameters in FMF patients did not differe by gender. Although C-reactive protein levels were within normal ranges, they were statistically higher than in the control group [1.62 (0.59$4.59)$ vs. 0.80 (0.47-1.72), $p=0.012$ ]. All other biochemical and transthoracic echocardiographic parameters were comparable between the two groups (Table 2).

The resting ECGs of all individuals were in sinus rhythm. Although corrected QT intervals were similar between the two groups, ventricular repolarization parameters [Tp-e $(86.91 \pm 16.49$ vs. $70.20 \pm 5.02, p<0.001)$ and Tp-e/QTc ratio $(0.21 \pm 0.04$ vs. $0.17 \pm 0.02, p<0.001)]$ were significantly prolonged in FMF patients (Table 1).

\begin{tabular}{|c|c|c|c|}
\hline & FMF group $(n=61)$ & Control group $(n=54)$ & $\mathrm{p}$ \\
\hline Age, years, mean $\pm S D$ & $34.3 \pm 10.0$ & $34.7 \pm 9.3$ & 0.838 \\
\hline Sex, male, n (\%) & $18(29.5)$ & $21(38.9)$ & 0.288 \\
\hline $\mathrm{BMI}, \mathrm{kg} / \mathrm{m}^{2}$, mean $\pm \mathrm{SD}$ & $24.8 \pm 2.4$ & $25.6 \pm 2.8$ & 0.144 \\
\hline Smoking, n (\%) & $18(29.5)$ & $14(25.9)$ & 0.670 \\
\hline $\mathrm{EF}, \%$, mean $\pm \mathrm{SD}$ & $62.3 \pm 3.0$ & $61.9 \pm 4.5$ & 0.620 \\
\hline Sedimentation rate, $\mathrm{mm} / \mathrm{h}$, median (IQR) & $14.00(6.00-21.50)$ & $11.00(8.75-14.00)$ & 0.154 \\
\hline C-reactive protein, mg/L, median (IQR) & $1.62(0.59-4.59)$ & $0.80(0.47-1.72)$ & 0.012 \\
\hline Sodium, mmol/L, median (IQR) & $138.00(137.00-140.00)$ & $138.00(137.00-140.00)$ & 0.826 \\
\hline Potassium, mmol/L, mean \pm SD & $4.23 \pm 0.39$ & $4.20 \pm 0.43$ & 0.781 \\
\hline $\mathrm{TSH}, \mathrm{mU} / \mathrm{L}, \mathrm{mean} \pm \mathrm{SD}$ & $1.27 \pm 0.77$ & $1.34 \pm 0.81$ & 0.885 \\
\hline Heart rate, beats/min, median (IQR) & $78.0(73.0-89.0)$ & $75.5(72.0-82.3)$ & 0.103 \\
\hline QRS interval, ms, mean $\pm S D$ & $78.34 \pm 9.65$ & $75.50 \pm 9.01$ & 0.130 \\
\hline QTc interval, ms, mean \pm SD & $414.72 \pm 20.12$ & $406.92 \pm 31.23$ & 0.122 \\
\hline Tpe, ms, mean $\pm S D$ & $86.91 \pm 16.49$ & $70.20 \pm 5.02$ & $<0.001$ \\
\hline Tpe/QTc ratio, mean $\pm S D$ & $0.21 \pm 0.04$ & $0.17 \pm 0.02$ & $<0.001$ \\
\hline
\end{tabular}




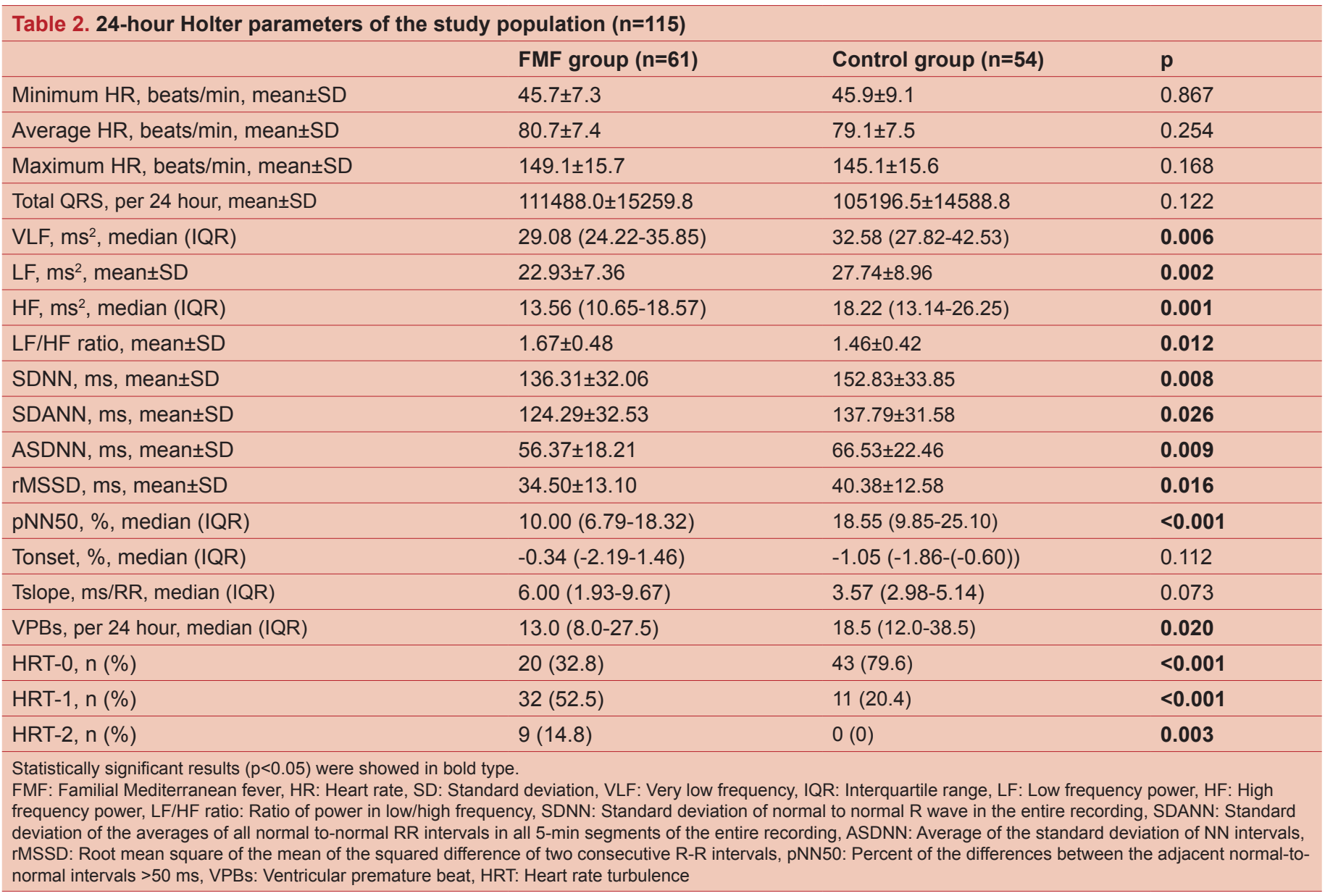

While the minimum-maximum-mean heart rates and total QRS were similar between the two groups, all other HRV parameters, including SDNN (136.31 \pm 32.06 vs. $152.83 \pm 33.85$, $p=0.008)$, SDANN (124.29 \pm 32.53 vs. $137.79 \pm 31.58, p=0.026)$, rMSSD (34.50 \pm 13.10 vs. $40.38 \pm 12.58, p=0.016), \quad p N N 50$ [10.00 (6.79-18.32) vs. $18.55(9.85-25.10), p<0.001], \quad$ VLF [29.08 (24.22-35.85) vs. 32.58 (27.82-42.53), $p=0.006]$, LF (22.93 \pm 7.36 vs. $27.74 \pm 8.96, p=0.002)$, HF [13.56 (10.65-18.57) vs. $18.22(13.14-26.25), p=0.001]$ were significantly lower in the FMF group. Compared to the control group, the LF/HF ratio $(1.67 \pm 0.48$ vs. $1.46 \pm 0.42, p=0.012)$ was significantly higher (Table 2).

HRT parameters were normal in $20(32.8 \%)$ of the FMF patients and $43(79.6 \%)$ of the control subjects. Thirty-two $(52.5 \%)$ patients were in the HRT-1 group (FMF vs. control; $32 / 61$ vs. $11 / 54, p<0.001)$, whereas $9(14.8 \%)$ patients were in the HRT-2 group ( $9 / 61$ vs. $0 / 54, p=0.003)$ in the FMF group (Table 2).

There was a moderate negative correlation between disease duration and HRV parameters and a moderate positive correlation between Tp-e and Tp-e/QTc ratio (Table 3). No significant difference was observed in resting ECG, HRV, and HRT parameters with regards to the mutations.
There were no significant differences in terms of demographic characteristics, Tp-e, and QTc intervals between anakinra-treated and non-anakinra-treated patients. Tp-e/QTc ratio $(0.24 \pm 0.05$ vs. $0.21 \pm 0.04, p=0.041$ ) was prolonged in patients using anakinra (Table 4). Both groups had similar minimum-mean-maximum heart rates, total $\mathrm{QRS}$, and ventricular extrasystole counts. All HRV variables VLF $(22.45 \pm 5.45$ vs. $31.42 \pm 8.17, \mathrm{p}=0.004)$, LF (17.02 \pm 4.99 vs. $23.82 \pm 7.28, p=0.014)$, HF $(10.42 \pm 3.42$ vs. $15.67 \pm 6.71, p=0.035)$, SDNN (114.00 \pm 15.60 vs. $139.67 \pm 32.63$, $\mathrm{p}=0.034)$, SDANN (87.50 \pm 12.50 vs. $129.84 \pm 30.99, p<0.001)$, ASDNN (39.75 \pm 13.84 vs. $58.88 \pm 17.54, p=0.005), \quad r M S S D$ (26.12 \pm 10.41 vs. $35.77 \pm 13.07, p=0.039)$, pNN50 [7.04 (1.20$8.12)$ vs. 11.10 (7.55-18.70), $p<0.001]$ were significantly lower in anakinra-treated patients than in non-anakinra-treated patients. Additionally, all patients using anakinra had abnormal HRT values (Table 5).

\section{Discussion}

This study found that Tp-e, Tp-e/QTc ratios were prolonged, and HRV and HRT parameters were impaired in patients with FMF versus the control group. Additionally, disease duration and anakinra treatment were associated with the impairment of these parameters. 
Table 3. Correlation analysis of disease duration, Tpe, Tpe/QTc and 24-hour Holter parameters of FMF patients

$\begin{array}{lllllllllll}\begin{array}{l}\text { Disease } \\ \text { duration, } \\ \text { years }\end{array} & \begin{array}{l}\text { Tpe, } \\ \text { msn }\end{array} & \begin{array}{l}\text { Tpe/QTc } \\ \text { ratio }\end{array} & \begin{array}{l}\text { VLF, } \\ \mathrm{ms}^{2}\end{array} & \begin{array}{l}\text { LF, } \\ \mathrm{ms}^{2}\end{array} & \begin{array}{l}\mathrm{HF}, \\ \mathrm{ms}^{2}\end{array} & \begin{array}{l}\text { SDNN, } \\ \mathrm{ms}\end{array} & \begin{array}{l}\text { SDANN, } \\ \mathrm{ms}\end{array} & \begin{array}{l}\text { ASDNN, } \\ \mathrm{ms}\end{array} & \begin{array}{l}\text { rMSSD, } \\ \mathrm{ms}\end{array} & \begin{array}{l}\text { pNN50, } \\ \%\end{array} \\ \end{array}$

Disease duration,

years

\begin{tabular}{|c|c|c|c|c|c|c|c|c|c|c|c|}
\hline$R$ & - & 0.402 & 0.382 & -0.370 & -0.304 & -0.319 & -0.544 & -0.398 & -0.314 & -0.304 & -0.332 \\
\hline$P$ & - & 0.001 & 0.003 & 0.004 & 0.020 & 0.012 & $<0.001$ & 0.002 & 0.014 & 0.017 & 0.009 \\
\hline \multicolumn{12}{|c|}{ Tpe, msn } \\
\hline$P$ & 0.001 & - & $<0.001$ & 0.001 & 0.003 & 0.002 & $<0.001$ & 0.014 & 0.001 & 0.031 & 0.002 \\
\hline \multicolumn{12}{|c|}{ Tpe/QTc ratio } \\
\hline
\end{tabular}

Tpe: Transmural dispersion of repolarization, QTc: Corrected QT interval, FMF: Familial Mediterranean fever, VLF: Very low frequency, LF: Low frequency power, HF: High frequency power, SDNN: Standard deviation of normal to normal R wave in the entire recording, SDANN: Standard deviation of the averages of all normal to-

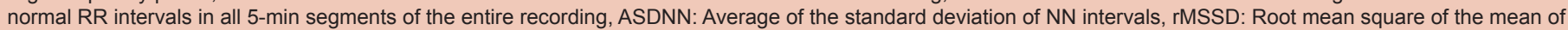
the squared difference of two consecutive R-R intervals, pNN50: Percent of the differences between the adjacent normal-to-normal intervals $>50$ ms

\begin{tabular}{|c|c|c|c|}
\hline & Anakinra group $(n=8)$ & Non-anakinra group $(n=53)$ & $\mathbf{p}$ \\
\hline Age, years, mean $\pm S D$ & $34.5 \pm 7.6$ & $34.3 \pm 10.4$ & 0.971 \\
\hline Sex, male, n (\%) & $4(50)$ & $14(26.4)$ & 0.218 \\
\hline $\mathrm{BMI}, \mathrm{kg} / \mathrm{m}^{2}$, mean $\pm \mathrm{SD}$ & $24.2 \pm 2.5$ & $24.9 \pm 2.4$ & 0.484 \\
\hline Smoking, $\mathrm{n}(\%)$ & $3(37.5)$ & $15(28.3)$ & 0.677 \\
\hline Disease duration, years, median (IQR) & $5.8(3.5-15.0)$ & $7.0(3.0-10.0)$ & 0.468 \\
\hline $\mathrm{EF}, \%$, mean $\pm \mathrm{SD}$ & $62.6 \pm 3.9$ & $62.2 \pm 2.9$ & 0.743 \\
\hline Sedimentation rate, $\mathrm{mm} / \mathrm{h}$, median (IQR) & $9.00(3.75-20.25)$ & $14.00(6.50-22.00)$ & 0.312 \\
\hline C-reactive protein, $\mathrm{mg} / \mathrm{L}$, median (IQR) & $1.18(0.30-6.53)$ & $1.66(0.60-4.60)$ & 0.777 \\
\hline Heart rate, beats/min, mean $\pm S D$ & $75.2 \pm 13.5$ & $81.9 \pm 10.4$ & 0.214 \\
\hline QRS interval, ms, mean $\pm S D$ & $85.62 \pm 14.59$ & $77.24 \pm 8.33$ & 0.151 \\
\hline QTc interval, ms, mean \pm SD & $405.62 \pm 14.36$ & $416.09 \pm 20.61$ & 0.172 \\
\hline Tpe, ms, mean \pm SD & $96.50 \pm 19.08$ & $85.47 \pm 15.77$ & 0.084 \\
\hline Tpe/QTc ratio, mean \pm SD & $0.24 \pm 0.05$ & $0.21 \pm 0.04$ & 0.041 \\
\hline
\end{tabular}

Statistically significant results $(p<0.05)$ were showed in bold type.

FMF: Familial Mediterranean fever, SD: Standard deviation, BMI: Body mass index, IQR: Interquartile range, EF: Ejection fraction, QTc: Corrected QT interval, Tpe: Transmural dispersion of repolarization

Cardiovascular events are the leading causes of death and morbidity in FMF and many other autoimmune diseases (4). Cardiac autonomic dysfunction is associated with ventricular arrhythmias and sudden death (18). Conduction disorders and arrhythmias are common symptoms of adverse cardiac outcomes in autoinflammatory diseases such as FMF (19). Cardiac autonomic dysfunction related to amyloidosis has been observed in patients with FMF (20). However, some studies have shown that patients with FMF without complications (without amyloidosis) also develop autonomic dysfunction (21). Moog et al. (22) stated that patients with FMF have impaired pupillomotor function which is controlled by the parasympathetic nervous system. Cardiac autonomic dysfunction (reduced parasympathetic dominance) has also been observed in many other autoimmune diseases, and it has been associated with disease activity and poor prognosis (23). The 24-hour Holter ECG monitoring, which is a cost-effective, reliable, and easily accessible method, is commonly used to evaluate cardiac ANS. HRV and HRT parameters obtained automatically from 24-hour Holter recordings are useful in evaluating cardiac ANS $(24,25)$. Some HRV parameters were depressed in diseases such as diabetes mellitus, hypertension, myocardial infarction, heart failure, chronic obstructive pulmonary disease, obstructive sleep apnea syndrome, and hypothyroidism (26). It was also reported that decreased HRV parameters could predict the mortality risk in heart failure and myocardial infarction (26). Depressed HRV parameters and increased LF/HF ratio are predictors of decreased parasympathetic dominance and increased cardiac 


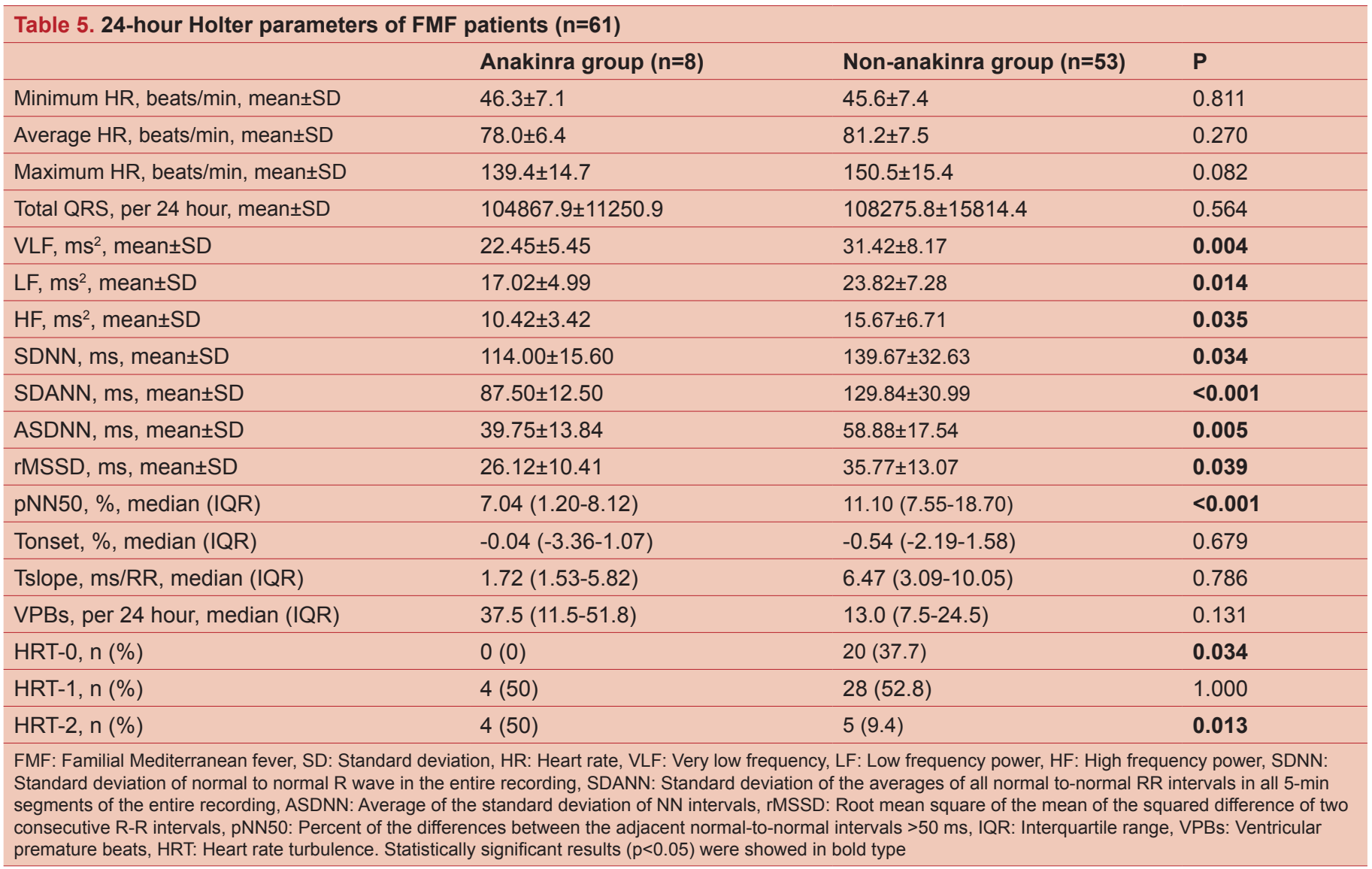

sympathetic dominance (25). If the parasympathetic nervous system's dominance in the heart weakens, arrhythmias are unavoidable (27). It has been shown that the evaluation of only one of the HRV parameters does not provide reliable information on the cardiac ANS. Therefore, it is important to evaluate all HRV parameters together (26). Canpolat et al. (28) found that many HRV parameters were impaired in FMF patients without amyloidosis. In our study, as in the literature, both time-domain (SDNN, SDANN, pNN50, rMSSD) and frequency domain (VLF, LF, HF) parameters decreased, while the LF/HF ratio increased in patients with FMF compared to the control group. Furthermore, disease duration was associated with the impairment of these parameters.

HRT, a more specific and sensitive method than HRV, provides data on cardiac ANS (29). Additionally, HRT gives information on the baroreceptor response (30). Impaired HRT was associated with arrhythmias and sudden death in patients with heart failure and myocardial infarction (31). HRT parameters were found to be impaired in chronic inflammatory disorders such as systemic sclerosis and systemic lupus erythematosus $(32,33)$. There are two separate variables in the HRT analysis; TO and TS. Cardiovascular deaths are higher among patients with impaired TO and TS (34). In our study, most patients with FMF had impairment in HRT variables (67.5\%).
Tp-e interval and Tp-e/QTc ratio are cost-effective and reliable parameters that can be used to quickly evaluate the transmural repolarization dispersion of the myocardium using a resting ECG (35). Impairment of these parameters may predict ventricular arrhythmias and sudden cardiac death. Also, these parameters can be used to assess cardiac risk in various diseases and the general population (35). It has been reported that a prolonged Tp-e interval may be a predictor of the cardiovascular mortality risk in inflammatory diseases (36). Some studies showed that ventricular repolarization parameters were impaired in patients with FMF compared to the healthy group $(37,38)$. In our study, we found that the Tp-e interval and Tp-e/QTc ratio were significantly longer in FMF patients than those in the control group. Moreover, we determined that these variables were correlated with disease duration and HRV parameters.

Colchicine is the cornerstone of FMF treatment. However, in colchicine-resistant cases, anakinra, the IL-1 receptor antagonist, is recommended, but its long-term effect on mortality is still unknown (39). Few studies have examined the relationship between anakinra use and cardiac arrhythmias. It has also been reported that anakinra may prevent arrhythmias after myocardial infarction (40). Conversely, in our study, we found that all HRV and HRT parameters were significantly impaired in patients using anakinra compared to those who did not use it. While 
there was no significant difference in Tp-e values, we found a significant impairment in the Tp-e/QTc ratio in anakinra-treated patients. Although the small number of patients using anakinra, this is the first study to evaluate 24-hour Holter and ECG in these patients. Patients who were using anakinra were more likely to have uncontrolled chronic inflammation for a longer period. This may explain why abnormal cardiac autonomic functions in these patients were more pronounced.

Our study has shown that patients with FMF may have autonomic dysfunction even if they do not have any cardiovascular symptoms. Therefore, patients with FMF should be constantly monitored for cardiovascular complications, particularly arrhythmias. It is difficult to perform 24-hour Holter ECG on all patients, however, Tp-e, a basic ECG measurement that correlates with 24-hour Holter ECG parameters, can be evaluated in daily practice. ECG should, in our opinion, be performed on patients who have been following up with FMF for a long period.

The higher number of patients, evaluating the relationship between resting ECG and 24-hour Holter ECG parameters in patients with FMF, and evaluating 24-hour Holter ECG recordings in patients using anakinra are the strengths of our study. Our study has some limitations. Since the study was designed retrospectively, the contribution of the impaired cardiac autonomic system to cardiovascular adverse events could not be evaluated in the long-term follow-up. Since some common comorbidities (such as hypertension, diabetes mellitus ...) were excluded from the study, our results may not be representative of the real world. Although there were no statistically significant differences in any parameters based on gender, the higher proportion of females is considered a limitation. Additionally, the number of colchicine-resistant cases treated with anakinra was low.

\section{Conclusion}

In conclusion, the ventricular repolarization, HRV, and HRT parameters were impaired in parallel with the disease duration in patients with FMF without complications compared to the control group. This impairment was common in anakinra-treated patients. Therefore, cardiac autonomic system dysfunction and arrhythmias, which may increase morbidity and mortality in patients with FMF, can be evaluated using easy and non-invasive methods such as standard ECG and 24-hour Holter ECG. On the other hand, studies with larger sample size and a long-term follow-up are required to further confirm these findings.

\section{Ethics}

Ethics Committee Approval: The study was approved by the Local Ethical Committee (Ethical Committee of Yozgat Bozok University, approval no: 2017-KAEK-189_2021.04.28_10).
Informed Consent: Retrospective study.

Peer-review: Externally peer-reviewed.

\section{Authorship Contributions}

Concept: M.C., B.C., Design: M.C., B.S., S.G.N., Data Collection or Processing: B.C., S.G.N., Analysis or Interpretation: M.C., B.C., Literature Search: M.C., B.S., Writing: B.S., M.C., B.C.

Conflict of Interest: No conflict of interest was declared by the authors.

Financial Disclosure: The authors declared that this study received no financial support.

\section{References}

1. Alghamdi M. Familial Mediterranean fever, review of the literature. Clin Rheumatol. 2017;36:1707-1713.

2. Booty MG, Chae JJ, Masters SL, et al. Familial Mediterranean fever with a single MEFV mutation: where is the second hit? Arthritis Rheum. 2009;60:1851-1861.

3. Ozen S. Changing concepts in familial Mediterranean fever: is it possible to have an autosomal-recessive disease with only one mutation? Arthritis Rheum. 2009;60:1575-1577.

4. Alsarah A, Alsara O, Laird-Fick HS. Cardiac manifestations of Familial Mediterranean fever. Avicenna J Med. 2017;7:158163.

5. Ozen S, Demirkaya E, Erer B, et al. EULAR recommendations for the management of familial Mediterranean fever. Ann Rheum Dis. 2016;75:644-651.

6. Belkhir R, Moulonguet-Doleris L, Hachulla E, Prinseau J, Baglin A, Hanslik T. Treatment of familial Mediterranean fever with anakinra. Ann Intern Med. 2007;146:825-826.

7. Iwasa A, Hwa M, Hassankhani A, Liu T, Narayan SM. Abnormal heart rate turbulence predicts the initiation of ventricular arrhythmias. Pacing Clin Electrophysiol. 2005;28:1189-1197.

8. Poliwczak AR, Waszczykowska E, Dziankowska-Bartkowiak B, Dworniak-Pryca K. Abnormalities of heart rate turbulence and heart rate variability as indicators of increased cardiovascular risk in patients with systemic sclerosis. Postepy Dermatol Alergol. 2019;36:707-713.

9. Whayne TF Jr, Morales GX, Darrat YH. Clinical Aspects of Systemic Inflammation and Arrhythmogenesis, Especially Atrial Fibrillation. Angiology. 2018;69:281-285.

10. Dregan A, Chowienczyk P, Molokhia M. Cardiovascular and type 2 diabetes morbidity and all-cause mortality among diverse chronic inflammatory disorders. Heart. 2017;103:18671873.

11. Lahiri MK, Kannankeril PJ, Goldberger JJ. Assessment of autonomic function in cardiovascular disease: physiological basis and prognostic implications. J Am Coll Cardiol. 2008;51:1725-1733.

12. Morin DP, Saad MN, Shams OF, et al. Relationships between the T-peak to T-end interval, ventricular tachyarrhythmia, and death in left ventricular systolic dysfunction. Europace. 2012;14:1172-1179. 
13. Porthan $\mathrm{K}$, Viitasalo $\mathrm{M}$, Toivonen $\mathrm{L}$, et al. Predictive value of electrocardiographic T-wave morphology parameters and T-wave peak to T-wave end interval for sudden cardiac death in the general population. Circ Arrhythm Electrophysiol. 2013;6:690-696.

14. Nussinovitch $\mathrm{N}$, Livneh $\mathrm{A}$, Katz $\mathrm{K}$, et al. Heart rate variability in familial Mediterranean fever. Rheumatol Int. 2011;31:39-43.

15. Kaya H, Süner A, Köroğlu S, Akçay A, Türkbeyler İH, Köleoğlu M. Heart rate variability in familial Mediterranean fever patients. Eur J Rheumatol. 2014;1:58-61.

16. No authors listed. Heart rate variability. Standards of measurement, physiological interpretation, and clinical use. Task Force of the European Society of Cardiology and the North American Society of Pacing and Electrophysiology. Eur Heart J. 1996;17:354-381.

17. Bauer A, Malik M, Schmidt G, et al. Heart rate turbulence: standards of measurement, physiological interpretation, and clinical use: International Society for Holter and Noninvasive Electrophysiology Consensus. J Am Coll Cardiol. 2008;52:1353-1365.

18. Wu P, Vaseghi $M$. The autonomic nervous system and ventricular arrhythmias in myocardial infarction and heart failure. Pacing Clin Electrophysiol. 2020;43:172-180.

19. Yorgun H, Canpolat U, Aytemir K, et al. Evaluation of cardiac autonomic functions in patients with systemic lupus erythematosus. Lupus. 2012;21:373-379.

20. Nussinovitch $U$, Volovitz B, Nussinovitch $M$, et al. Abnormal heart rate variability in $A A$ amyloidosis of familial Mediterranean fever. Amyloid. 2011;18:206-210.

21. Rozenbaum M, Naschitz JE, Yudashkin M, et al. Cardiovascular autonomic dysfunction in familial Mediterranean fever. J Rheumatol. 2002;29:987-989.

22. Moog P, Valda K, Eren OE, et al. A complex pattern of autonomic dysfunction in familial Mediterranean fever. Results from a controlled cross-sectional study. Clin Exp Rheumatol. 2019;37(Suppl 121):35-42. Available from: file:///Users/ galenos/Downloads/article.pdf

23. Thanou A, Stavrakis S, Dyer JW, Munroe ME, James JA, Merrill JT. Impact of heart rate variability, a marker for cardiac health, on lupus disease activity. Arthritis Res Ther. 2016;18:197.

24. Cygankiewicz I. Heart rate turbulence. Prog Cardiovasc Dis. 2013;56:160-171.

25. Cygankiewicz I, Zareba W. Heart rate variability. Handb Clin Neurol. 2013;117:379-393.

26. Kleiger RE, Stein PK, Bigger JT Jr. Heart rate variability: measurement and clinical utility. Ann Noninvasive Electrocardiol. 2005;10:88-101.

27. Kalla M, Herring N, Paterson DJ. Cardiac sympathovagal balance and ventricular arrhythmia. Auton Neurosci. 2016;199:29-37.
28. Canpolat U, Dural M, Aytemir K, et al. Evaluation of various cardiac autonomic indices in patients with familial Mediterranean fever on colchicine treatment. Auton Neurosci. 2012;167:70-74.

29. Lin K, Wei L, Huang Z, Zeng Q. Combination of Ewing test, heart rate variability, and heart rate turbulence analysis for early diagnosis of diabetic cardiac autonomic neuropathy. Medicine. 2017;96:e8296.

30. Cygankiewicz I, Wranicz JK, Bolinska H, Zaslonka J, Zareba W. Relationship between heart rate turbulence and heart rate, heart rate variability, and number of ventricular premature beats in coronary patients. J Cardiovasc Electrophysiol. 2004;15:731-737.

31. Disertori M, Mase M, Rigoni M, Nollo G, Ravelli F. Heart Rate Turbulence Is a Powerful Predictor of Cardiac Death and Ventricular Arrhythmias in Postmyocardial Infarction and Heart Failure Patients: A Systematic Review and Meta-Analysis. Circ Arrhythm Electrophysiol. 2016;9:e004610.

32. Bienias $P$, Ciurzynski M, Glinska-Wielochowska $M$, et al. Heart rate turbulence assessment in systemic sclerosis: the role for the detection of cardiac autonomic nervous system dysfunction. Rheumatology (Oxford). 2010;49:355-360.

33. Poliwczak AR, Waszczykowska E, Dziankowska-Bartkowiak $B$, Kozirog M, Dworniak K. The use of heart rate turbulence and heart rate variability in the assessment of autonomic regulation and circadian rhythm in patients with systemic lupus erythematosus without apparent heart disease. Lupus. 2018;27:436-444.

34. Stein PK, Deedwania P. Usefulness of abnormal heart rate turbulence to predict cardiovascular mortality in high-risk patients with acute myocardial infarction and left ventricular dysfunction (from the EPHESUS study). Am J Cardiol. 2009;103:1495-1499.

35. Tse G, Gong M, Wong WT, et al. The Tpeak - Tend interval as an electrocardiographic risk marker of arrhythmic and mortality outcomes: A systematic review and meta-analysis. Heart Rhythm. 2017; 14:1131-1137.

36. Fidanci K, Gulgun M, Demirkaya E, et al. Assessment of autonomic functions in children with familial Mediterranean fever by using heart rate variability measurements. Int J Rheum Dis. 2017;20:2086-2092.

37. Ahbap E, Sakaci T, Kara E, et al. Familial Mediterranean Fever is associated with abnormal ventricular repolarization indices. Rev Med Chil. 2015;143:1560-1568.

38. Karaman K, Karayakali M, Erken E, et al. Assessment of myocardial repolarisation parameters in patients with familial Mediterranean fever. Cardiovasc J Afr. 2017;28:154-158.

39. Demirkaya E, Erer B, Ozen S, Ben-Chetrit E. Efficacy and safety of treatments in Familial Mediterranean fever: a systematic review. Rheumatol Int. 2016;36:325-331.

40. De Jesus NM, Wang L, Lai J, et al. Antiarrhythmic effects of interleukin 1 inhibition after myocardial infarction. Heart Rhythm. 2017; 14:727-736. 